IAU Colloquium 164: Radio Emission from Galactic and Extragalactic Compact Sources

ASP Conference Series, Vol. 144, 1998

J. A. Zensus, G. B. Taylor, \& J. M. Wrobel (eds.)

\title{
EVN+MERLIN Observations of 3C 66B and a Two-Fluid Model for VLBI Jets
}

D. Fraix-Burnet

Observatoire de Grenoble, BP 53, F-38041 Grenoble Cédex 9, France

V. Despringre

Observatoire Midi-Pyrénées, F-31400 Toulouse, France

A. Baudry

Observatoire de Bordeaux, BP 89, F-33270 Floirac, France

\begin{abstract}
We present observations of the FR I radiogalaxy $3 \mathrm{C} 66 \mathrm{~B}$ made during a joint EVN + MERLIN VLBI session in May 1995. The jet is mapped from the 5 mas scale up to about 3 arcmin. The newly mapped VLBI jet shows essentially two elongated blobs in the direction of the brightest kpc jet (NE). We also present the first results obtained for a two-fluid model for VLBI jets.
\end{abstract}

\section{Observations of $3 \mathrm{C} 66 \mathrm{~B}$}

The observations took place on May 21st, 1995, during a joint EVN and MERLIN session. Six EVN antennas (Effelsberg, Jodrell, Onsala, Medicina, Noto, Simeiz) plus MERLIN and Westerbork arrays provide the first mapping of $3 \mathrm{C} 66 \mathrm{~B}$ from a few mas up to several arcmin.

Four maps were made (Fig. 1; Despringre, Fraix-Burnet, \& Baudry 1997). The WSRT array provides a map of the whole radiogalaxy with a resolution of 5 arcsec. The MERLIN map at a resolution of 150 mas shows an unresolved core together with the brightest component of the jet, i. e. knot $B$ at 2.9 arcsec from the nucleus. An over-resolved MERLIN map at a resolution of 20 mas shows a NE 35 mas $(\simeq 15 \mathrm{pc})$ extension in the nucleus.

This extension shows up as a distinct component on the EVN + MERLIN map (resolution of 5 mas) and is not seen on an EVN-only map. There is another component closer to the nucleus $(15$ mas $\simeq 6 \mathrm{pc}$ ) which points exactly toward the direction of the large scale jet. By fitting elliptical gaussians to the three components (the core and the two blobs at 15 and 35 mas), flux densities are found to be respectively 180,11 and $3 \mathrm{mJy}$.

\section{A Two-Fluid Model for VLBI Jets}

An interesting alternative to the relativistic shocked jet model is the two-fluid concept (Sol, Pelletier, \& Asséo 1989):

- the bulk of the jet (electrons and protons ejected from the accretion disk in the form of a collimated wind) is non- or mildly-relativistic at all scales

- synchrotron radiation and relativistic phenomena are produced by a beam of relativistic electrons/positrons.

In our model for VLBI jets, the jet itself is non- or mildly-relativistic (electrons and protons), while the radiating blobs are relativistic electron-positron 


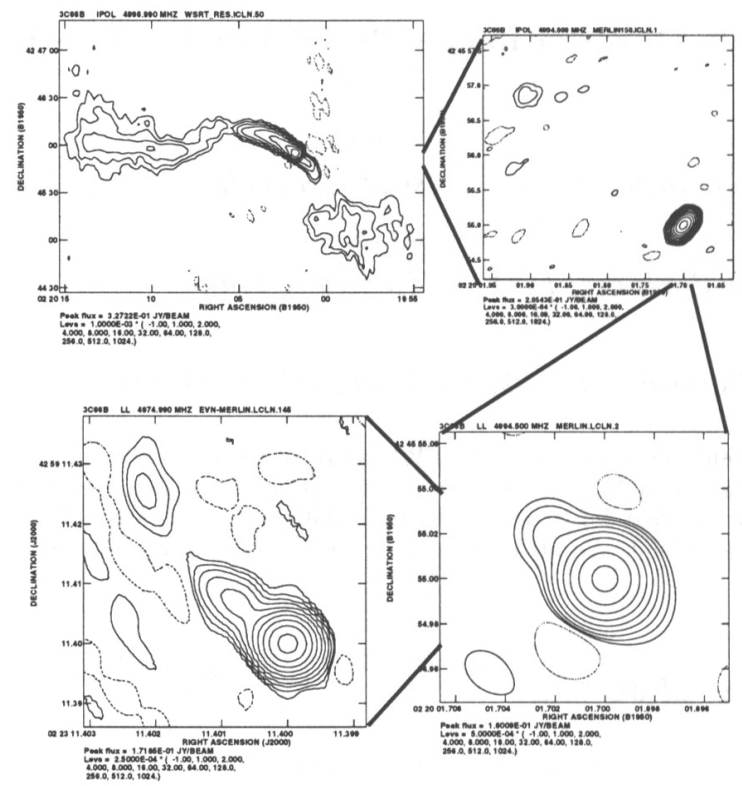

Figure 1.

"clouds" moving on helical paths wrapped around the jet. The term cloud is defined here as an ensemble of relativistic particles occupying a limited region of the jet, but these particles and the jet plasma are fully interpenetrated, making a two-component plasma.

In this work, the emphasis is on the physical description of the clouds, and not on the structure or origin of the trajectory. In the simple case where the magnetic field is uniform and homogeneous across the cloud, and the properties of the cloud are constant, synthetic maps of VLBI jets in different configurations are computed, as well as the variation of different observational parameters along the trajectory. Details and results are presented in Despringre \& FraixBurnet (1997). A turbulent magnetic will be included (Fraix-Burnet \& Vigon, in preparation), and the temporal physical evolution of the "clouds" from $10^{-3} \mathrm{pc}$ up to about one pc will be the most interesting part of this study. Our model is already consistent with the slight oscillation suggested by Fig. 1 for the VLBI 35 mas blob along the jet of $3 \mathrm{C} 66 \mathrm{~B}$.

\section{References}

Despringre, V., \& Fraix-Burnet, D. 1997. $A \& A, \mathbf{3 2 0}, 26-32$.

Fraix-Burnet, D., Despringre, V., \& Baudry, A. 1997. Vistas in Astronomy, (special issue), eds. M. Garrett \& F. Colomer, 41, 239-240.

Sol, H., Pelletier, G., \& Asséo, E. 1989. MNRAS, 237, 411-429. 